\title{
Challenges in Human-Centered Information Visualization: Introduction to the Special Issue
}

\author{
Xia Lin, Andreas Kerren, and Jiajie Zhang \\ Guest Editors
}

Human-centered computing has been described as "an emerging field that aims at bridging the existing gaps between the various disciplines involved with the design and implementation of computing systems that support people's activities"1. Information visualization is certainly one of the disciplines in the center of human-centered computing ${ }^{2}$. Or is it? Since we announced the Call for Paper for a special issue on human-centered information visualization, our authors and reviewers have been debating what should be called "Human-Centered Information Visualization" (HCIV). On one extreme, we might claim that all the research on information visualization is "human-centered" since the ultimate goal of information visualization is to let people understand and use the information presented visually. On the other extreme, we might regard the lack of "human-centered" approach as the major issue of current research in information visualization. In this special issue, through a set of excellent research papers selected by the reviewers, we seek to present the perspectives of information visualization researchers (the authors) on the issue of "human-centered" and hope to encourage more conversations on the advancement of information visualization toward "human-centered".

In the first paper, Measuring Effectiveness of Graph Visualizations: A Cognitive Load Perspective, Authors Huang et al. questioned the relationship between information visualization and human cognitive loads. They developed an interesting model to measure cognitive loads of graph visualizations and predict the regions of cognitive loads where visualization would be most effective. Their empirical results show promise of the model in revealing the relationship between visual understanding and domain complexity, data complexity, task complexity, and visual complexity.

In the second paper, What does the User Want to See? What does the Data Want to Be?, authors Pretorius and van Wijk argued that information visualization needs to be designed from two perspectives: the user's perspective and the data perspective. It is emphasized that the two perspectives are inter-related and reinforced mutually. They presented several case studies to demonstrate that it is important to consider not only user requirements but also the data to be visualized. In particular, switching from one perspective to another during the iterative design process would significantly benefit the design and the outcome. Their case studies provide some good lessons about building useful information visualization systems.

The third paper is about visual information filtering in the searching environment. Titled "Adaptive Visualization of Search Results: Bring User Models to Visual Analytics," the paper by Ahn and Brusilovsky introduces a framework that incorporates user modeling and information visualization to provide flexible and user-centered visual information filtering. In their system, retrieved documents are displayed at locations relatively to the point of interest (POI), which can be either user's query terms or terms generated through user modeling. The user can select to enact or disable a POI to filter through the retrieved documents. In their experimental testing, they specifically tested the user model effect on three 
different graphical layouts and evaluated how well user modeling helped to differentiate relevant and nonrelevant documents on the visual displays.

The fourth and fifth articles are about applying information visualization to practical problems in the real world. In the article Knowledge Generation through Human-centered Information Visualization, authors Einsfeld et al. address the issue of making information visualization techniques work for nonexperts in specific domains. They describe an application of information visualization in a 3D environment that allows non-experts to follow visual metaphors to understand semantic relationships of objects both in the virtual world and in the real world. Their results show that visualization helps the user gain better understanding of the data by connecting previously isolated items. Similarly, in the article Seeing is Believing: Linking Data with Knowledge, authors Dadzie et al. present a research project on the development of a visual semantic information space to support sense-making activities. They developed several "knowledge views" using an enhanced user-centered design process. Their user testing results indicated that users understand the values and semantics presented on the visual views and were able to use the visual views for complex reasoning tasks.

The sixth paper, Visual Analysis of Dynamic Data Streams by Chin, et al., described their prototyping activities to refine and generalize existing visualization techniques to work with real-time data streams. Five different visualization tools, including dynamic treeview, treemap, spiral timeline, graphview, and multi-resolution network, were developed. Experiments were conducted to test how these tools help user interact with dynamic data in different visual context such as hierarchies, categories, time, and geography.

Finally, we include a special report on the Visual Analytics Science and Technology (VAST) contests over the last three years. VAST contests provide real world data and tasks to let researchers apply their visualization tools to solve specific problems and compare their different approaches to the same tasks. The evaluation of the visualization and contest results is particularly focused on the end-user perspective. Thus, the contests have the effect on bringing information visualization more close to end-users and to real world problems. We believe that lessons learned from these contests would be valuable for all the information visualization researchers.

\section{References:}

[1] Jaimes, A.; Sebe, N.; Gatica-Perez, D. (2006). Human-Centered Computing: A Multimedia Perspective. Proceedings of the 14th annual ACM international conference on Multimedia (Santa Barbara, CA, USA), 855-864.

[2] Kerren, A.; Stasko, J. T.; Fekete, J.-D.; North, C. (2008). Information Visualization - HumanCentered Issues and Perspectives. Volume 4950 of LNCS State-of-the-Art Survey, Springer. 